\title{
Norio Kaifu
}

\author{
A pioneer of millimetre-wave astronomy, Norio Kaifu was an incomparable leader, person of spirit, colleague and \\ friend.
}

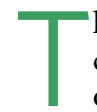
he international astronomy community is saddened by the death of Norio Kaifu, who passed away on 13 April 2019 after a three-year fight against pancreatic cancer. Leading major projects such as the $45-\mathrm{m}$ radio telescope at Nobeyama and the Subaru Telescope in Hawaii, he expanded the frontiers of millimetre-wave and optical-infrared astronomy.

Norio Kaifu started his career in astronomy in 1966 as a graduate student at the University of Tokyo, joining the radio astronomy group at its Tokyo Astronomical Observatory. Kenji Akabane and Masaki Morimoto were leading a group project constructing a millimetre-wave telescope of 6-m diameter. Inspired by the detection of interstellar water and ammonia molecules at wavelengths near $1 \mathrm{~cm}$ in the late 1960s, Norio recognized the importance of millimetre-wave spectroscopy: there would be numerous transitions of interstellar molecules in the millimetre wavelength that could be used to probe the yet undiscovered Universe. Appointed as assistant professor at the University of Tokyo in 1969, he started to develop radio spectrometers for the 6-m telescope, publishing the detection of methylamine $\left(\mathrm{CH}_{3} \mathrm{NH}_{2}\right)$, a precursor of amino acids, at $73 \mathrm{GHz}(4.1 \mathrm{~mm})$ in 1974 . He stayed at the National Radio Astronomy Observatory (NRAO) from 1972 to 1974 as a visiting research associate. Using the NRAO 11-m telescope at Kitt Peak, he detected a $\mathrm{SiO}$ emission at $86 \mathrm{GHz}$ from many $\mathrm{OH} /$ IR stars, confirming that the emission was a new type of maser.

In the 1970s, a plan for the Nobeyama 45-m diameter telescope and five-element interferometer for millimetre-wave astronomy was taking shape. Norio became a core member of the project and was appointed as associate professor in 1979. He was in charge of achieving the ambitious specifications of the $45-\mathrm{m}$ telescope: surface and tracking accuracies of $200 \mu \mathrm{m}$ (r.m.s.) and 4 arcsec (r.m.s.), respectively. However, above all, his unique and ingenious contribution to the project was the development of a state-of-the-art acousto-optical spectrometer. It had 16,000 channels covering a bandwidth of $2 \mathrm{GHz}$ at a time, with another set of 16,000 channels for simultaneous high-resolution spectroscopy. After the telescope was completed in

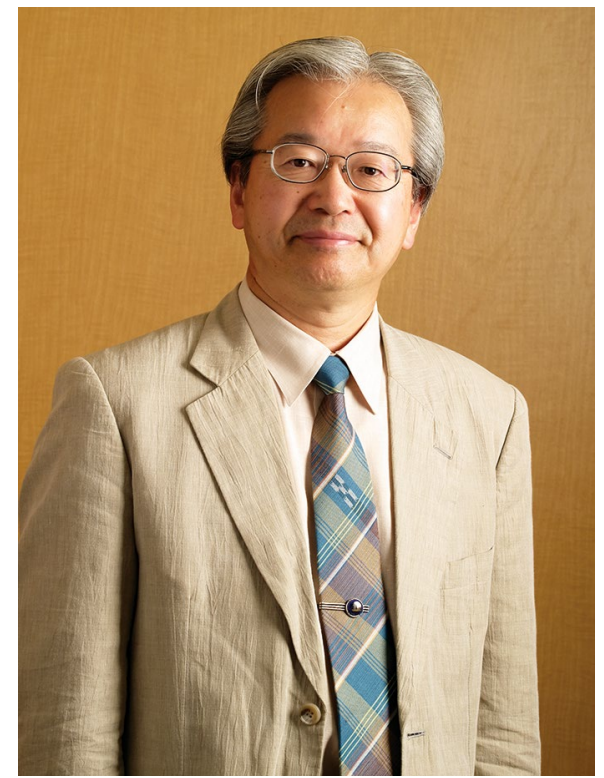

Norio Kaifu (1943-2019). Credit: NAOJ

1982, he led a group of astronomers and chemists to carry out surveys of spectral lines, discovering more than a dozen new, mostly organic, interstellar molecules. He also led studies of star formation, reporting for the first time a disk-like structure around a protostar and the hollow-cone nature of molecular outflows. The extremely highvelocity water maser emission from the centre of NGC 4258, the first clear evidence for the presence of a supermassive black hole at the centre of a galaxy, could not have been made without the wide-band capability of his spectrometer.

The success of the Nobeyama telescopes in the 1980s encouraged Japanese optical astronomers to build the largest-aperture optical telescope. To handle such a big project, the Tokyo Astronomical Observatory left the University of Tokyo in 1988 and formed the independent National Astronomical Observatory of Japan (NAOJ) together with two other organizations. Norio Kaifu became a full professor there. He was invited to join the Japanese National Large Telescope project in 1990. Considering ideas from the general public, he named the telescope Subaru, the ancient Japanese word for the Pleiades star cluster. He then led the instrumentation programme for Subaru, making the critical decision to develop a wide-field camera, later called the SuprimeCam, for its prime focus. Moving to Hawaii's Big Island, he served as the first director of the Subaru Telescope, an observatory under NAOJ established in 1997. The telescope was successfully inaugurated in 1999 and has since made numerous scientific achievements.

Norio Kaifu was one of the main champions for the internationalization of astronomy in Japan. When British astronomers became interested in the Nobeyama 45-m telescope in the early 1980s, he organized bilateral collaborations, including with the UKIRT (United Kingdom Infrared Telescope), then the world's largest infrared telescope. Quite a few astronomers from outside Japan visited Nobeyama, and those in Japan had the opportunity to visit Hawaii to make observations with the UKIRT. This collaboration was so fruitful not only because of its scientific outcomes, but because it provided opportunities for young Japanese astronomers to get acquainted with international researchers who would later play leading roles in world astronomy. Many of these Japanese astronomers eventually became the core members of the Subaru Project.

Norio often said that it was rather odd that astronomers in neighbouring regions of east Asia did not know each other and did not collaborate more closely together. In the 1980s, he started working with leading astronomers in China, South Korea and Taiwan to organize regional collaborations, guiding them to form in 2005 the East Asian Core Observatories Association (EACOA), an ongoing collaboration platform among the four major observatories in east Asia. A significant outcome of this is that Japan was able to team up with Taiwan, and now with South Korea as well, to become a partner of the Atacama Large Millimeter/submillimeter Array (ALMA). The East Asian Observatory was officially established under the EACOA in 2014.

Norio served as director general of NAOJ from 2000 to 2006. Aside from the major issue of Japan's participation in ALMA, he played a key role in the reorganization of NAOJ. In 2004, all national universities and inter-university research institutes in Japan were privatized. Using this opportunity, he restructured NAOJ to become an organization capable of carrying out big 
projects, especially international ones. Although NAOJ left the University of Tokyo in 1988, it had remained a university-style research and educational organization until this change. This meant that there were independent researcher groups led by individual professors who might voluntarily work together for projects like Subaru. The reorganization of NAOJ meant that projects came first as the main framework of the observatory, with researchers and other staff members located under each project, giving larger flexibility for projects to pursue their purposes. Norio retired from NAOJ in 2006. From 2005 to 2011, he served as a member of the Science Council of Japan, where he was pivotal in devising a masterplan for Japan's large projects in all scientific fields, including the humanities and social sciences.
He served as president of the International Astronomical Union from 2012 to 2015.

In addition to his research and administrative duties, Norio published more than 30 books for children and the public and was a regular book reviewer for the Japanese newspaper Mainichi Shimbun. His interests were diverse, including Japanese classic poetry and Asian mythology, but were always related to stars and the Universe. His life partner Shigemi was usually one of the first to read his books, giving him critical feedback.

Among the prizes he received were the Nishina Memorial Prize for the development of millimetre-wave astronomy in 1987, the Japan Academy Prize for research on the interstellar medium in 1998 and the Mainichi Book Review Prize in 2012.
He had been working actively until October last year, interested in astrobiology, dreaming of the next generation of extremely large telescopes, which will have the potential to detect biosignatures from exoplanets. When seen in his office at NAOJ last year, he said that he now felt at ease because he was aware of the limited amount of time remaining. His passing is a huge loss for the astronomy community in Japan and beyond.

Masahiko Hayashi

Japan Society for the Promotion of Science, Bonn, Germany.

e-mail:hayashi@jsps-bonn.de

Published online: 15 May 2019

https://doi.org/10.1038/s41550-019-0802-3 